\title{
Erratum zu: Subjektive Verarbeitungen von Aktivierung in prekären Lebenslagen
}

\author{
Carmen Figlestahler $\cdot$ Lisa Yashodhara Haller • Marko Tapio Perels • \\ Júlia Wéber · Franz Zahradnik • Mechthild Bereswill
}

Online publiziert: 25 . November 2015

(C) Springer Fachmedien Wiesbaden 2015

\section{Erratum zu: Soz Passagen \\ DOI 10.1007/s12592-015-0205-y}

Im ursprünglichen Beitrag war der Vorname von Herrn Franz Zahradnik falsch geschrieben. Weiterhin war der akademische Grad von Frau Júlia Wéber fälschlicherweise mit M.A. Germanistik angegeben. Beide Fehler wurden nun in diesem Erratum korrigiert.

Die Online-Version des Originalartikels ist unter doi:10.1007/s12592-015-0205-y zu finden.

Prof. Dr. phil. habil. M. Bereswill ( $₫)$

Institut für Sozialwesen, Soziologie sozialer Differenzierung \& Soziokultur, Universität Kassel,

Arnold-Bode-Straße 10,

34127 Kassel, Deutschland

E-Mail: bereswill@uni-kassel.de

C. Figlestahler, M.A. Soziologie

Kassel, Deutschland

E-Mail: c.figlestahler@uni-kassel.de

L. Y. Haller, Dipl. Pol.

Kassel, Deutschland

E-Mail: haller@uni-kassel.de

M. T. Perels, M.A. Sozialpsychologie/Soziologie

Kassel, Deutschland

E-Mail: perels@uni-kassel.de

J. Wéber, Dipl. Soz. Päd., Mag. deutsche Philologie

Institut für Erziehungswissenschaft, Technische Universität Berlin,

Marchstraße 23,

10587 Berlin, Deutschland

E-Mail: julia.weber@tu-berlin.de

F. Zahradnik, Dipl. Soziologe

Institut für Erziehungswissenschaft,

Freiestrasse 36,

Zürich CH-8032, Schweiz

E-Mail: franz.zahradnik@ife.uzh.ch 